\title{
Physio- und Ergotherapie können die Lebensqualität nicht verbessern
}

Fragestellung: Ob Ergotherapie und Physiotherapie klinisch wirksam und kosteneffizient sind, ist bisher nur wenig bearbeitet worden.

Hintergrund: Alle Therapien, die zur Behandlung der Parkinson-Erkrankung eingesetzt werden, müssen hinsichtlich der Kriterien Wirksamkeit und Kosteneffizienz aussagekräftig geprüft werden.

Patienten und Methodik: In die hier vorliegende Studie wurden in 38 Zentren in Großbritannien insgesamt 762 Patienten mit leichter bis mäßig schwerer Parkinson-Krankheit über einen Zeitraum von drei Jahren rekrutiert und jeweils 15 Monate lang nachbeobachtet. Das Einschlusskriterium war eine Einschränkung der Aktivitäten des täglichen Lebens, gemessen mit der ADL-Skala („activities of daily living“). Das Studiendesign war multizentrisch und offen mit einem Parallelgruppendesign.

Der Behandler musste unsi-

Clarke CE, Patel S, Ives $\mathrm{N}$ et al. Physiotherapy and occupational therapy versus no therapy in mild to moderate Parkinson's Disease. A randomized clinical trial (PD REHAB). JAMA Neurol 2016; 73: $291-9$ cher sein, ob der Patient in den nächsten 15 Monaten Physio- oder Ergotherapie benötigen wird.

Der Haupt-Outcome-Parameter war ein in der Rehabilitation genutzter Score, der „Nottingham Extended
ADL (NEADL)-Score“, der bei Studieneinschluss durch eine persönliche Untersuchung des behandelnden Arztes und anschließend brieflich nach drei, neun und 15 Monaten erhoben wurde. Auch alle anderen sekundären Outcome-Parameter waren Veränderungen in Selbstbeurteilungsbögen.

Ergebnisse: Im 15-Monats-Zeitraum erhielten 381 Patienten Physio- und Ergotherapie und ebenfalls 381 keine dieser Therapien. Die Behandlungsdauer betrug vier Therapiesitzungen von jeweils 58 Minuten Dauer während eines Zeitraums von acht Wochen.

Der Haupt-Outcome-Parameter (NEADL-Score) nach drei Monaten war in den beiden Gruppen nicht unterschiedlich. Auch die anderen sekundären Outcome-Parameter (Lebensqualität gemessen mit dem PDQ-39 bzw. dem EuroQol) waren grenzwertig oder negativ. Über den Nachbeobachtungszeitraum wies der NEADL-Score keine Veränderung auf und die Lebensqualitätsparameter zeigten bestenfalls minimale Unterschiede zugunsten der Therapie. Allerdings erhielten die Patienten in dem Jahr der Nachbeobachtung keine weitere Physio- oder Ergotherapie.

Schlussfolgerungen: Die Autoren folgern aus diesen Ergebnissen, dass Physio- und Ergotherapie nicht zu einer belangvollen Verbesserung der Lebensqualität oder der Aktivitäten des täglichen Lebens führen.

\section{- Kommentar von Günther Deuschl, Kiel}

\section{Meine Indikationsstellung wird durch diese Studie nicht beeinflusst}

Bei dieser Untersuchung handelt es sich um eine „pragmatische" Outcome-Studie, die als Nebenprodukt normaler klinischer Versorgung mit nur einem Kontakt mit den Studienärzten durchgeführt wurde. Die Studie hat bei Patientenorganisationen und in Therapeutenkreisen zu erheblicher Kritik geführt (z. B.http://www.apta.org/PTinMotion/News/2016/01/27/ PTPDIneffective/).

Tatsächlich kann man viele Einwände formulieren: Der Outcome-Parameter ist für die Parkinson-Krankheit kaum validiert, die Interventionen wurden nur untypisch kurz eingesetzt, im Nachbeobachtungszeitraum wurde nicht mehr therapiert und die Population wurde vorselektiert, weil Patienten, die gemäß Arzturteil in den nächsten 15 Monaten Physiotherapie brauchen werden, nicht eingeschlossen wurden. De facto wurde gezeigt, dass vier Sitzungen (Physio- oder Ergotherapie ohne Qualitätsvorgaben) à eine Stunde über einen Zeitraum von acht Wochen die Lebensqualität und Aktivitäten des Lebens von leicht bis mittelschwer betroffenen ParkinsonPatienten nicht verbessern.
Die bestehenden Widersprüche dieser Ergebnisse zur existierenden Datenlage lassen sich durch diese bedeutenden methodischen Schwächen weitgehend erklären.

Es ist wichtig zu wissen, dass es diese Studie gibt, weil sie zur Kostenbegrenzung sicher gerne zitiert wird. Viel mehr Studien gibt es aber, die zeigen, dass besonders Physio- und Ergotherapie bei Patienten mit Beeinträchtigung effektiv ist. Meine Indikationsstellung wird diese Studie nicht beeinflussen.

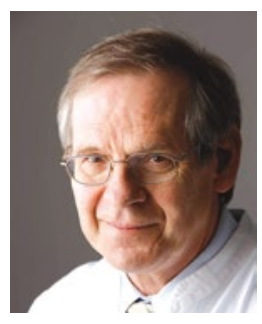

Prof. Dr. Dr. h.c. Günther Deuschl, Kiel

Emeritierter Direktor der Klinik für Neurologie, Universitätsklinikum Schleswig-Holstein, Campus Kiel (UKSH)

E-Mail: g.deuschl@neurologie.uni-kiel.de 